This article has been downloaded from IOPscience. Please scroll down to see the full text article. (http://iopscience.iop.org/0741-3335/49/12B/S26)

More related content is available

Download details:

IP Address: 128.178.125.67

The article was downloaded on 28/04/2008 at 11:17

Please note that terms and conditions apply. 


\title{
Statistical properties of electrostatic turbulence in toroidal magnetized plasmas
}

\author{
B Labit ${ }^{1}$, A Diallo ${ }^{1}$, A Fasoli ${ }^{1}$, I Furno ${ }^{1}$, D Iraji ${ }^{1}$, S H Müller ${ }^{1,3}$,

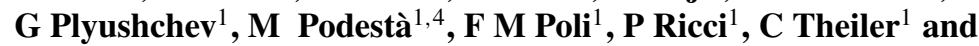 \\ J Horaček ${ }^{2}$ \\ ${ }^{1}$ Centre de Recherches en Physique des Plasmas (CRPP), Ecole Polytechnique Fédérale de \\ Lausanne (EPFL), Association Euratom-Suisse, CH-1015 Lausanne, Switzerland \\ ${ }^{2}$ Institute of Plasma Physics, Association EURATOM/IPP.CR, Praha, Czech Republic
}

Received 6 July 2007, in final form 2 September 2007

Published 16 November 2007

Online at stacks.iop.org/PPCF/49/B281

\begin{abstract}
A unique parabolic relation is observed to link skewness and kurtosis of density fluctuation signals, measured over the whole cross-section of the simple toroidal device TORPEX for a broad range of experimental conditions. This relationship is also valid for density fluctuation signals measured in the scrape-off layer of the TCV tokamak. All the probability density functions (PDFs) of the measured signals, including those characterized by a negative skewness, are universally described by a special case of the beta distribution. In TORPEX, fluctuations in the drift-interchange frequency range are necessary and sufficient to assure that PDFs can be described by this specific beta distribution. For a more detailed plasma scenario, it is shown that electron temperature and plasma potential fluctuations have different statistical properties compared with the density.
\end{abstract}

\section{Introduction}

The transport associated with plasma turbulence constitutes one of the main limiting factors for the performance of magnetic fusion devices. Fluctuations leading to a turbulent state and possible methods to control them have been investigated for many years [1]. A statistical description of plasma turbulence [2] is appropriate to capture some of the underlying physical mechanisms. Significant effort is dedicated to finding universal aspects in this statistical description [3], i.e. a common behavior for all spatial and temporal scales and/or for different physical systems and experimental conditions. The knowledge of some of these universal properties may lead to a better understanding of the impact of different

\footnotetext{
3 Present address: Center for Energy Research University of California of San Diego, La Jolla, CA-92093, USA.

4 Present address: University of California at Irvine, Irvine, CA-92697, USA.
} 
types of micro-instabilities on the macroscopic behavior of the plasma, e.g. in terms of fluctuation induced transport. In magnetic fusion plasmas, the universality of the probability density function (PDF) is investigated in various devices and experimental conditions, only using local density measurements at the plasma edge or in the scrape-off layer (SOL) (see [4] and references therein). Despite the common observation that PDFs of density fluctuations are non-Gaussian, there is no consensus on a unique PDF that could model all the experiments.

In this paper, we report on the statistical properties of the density fluctuations, in particular on their universal character, measured in the TORPEX toroidal device [5], in various experimental scenarios described in section 2. We first investigated the universality of the fluctuations by fitting the experimental PDFs with some analytical distributions evaluated with two experimental or optimized parameters. None of the tested distribution can universally fit the experimental data, section 3. Therefore, a method based on higher moments of the PDF is proposed. Such a method unambiguously reveals the universal character of the plasma density fluctuations: a second order polynomial links the third and fourth moments, section 4 . In section 5 , we show that the beta distribution, which has not yet been tested, fits properly the experimental data, reinforcing this universal character. To progress towards an identification of the physical mechanism behind the universality, the role of the drift-interchange frequency range is investigated in section 6. Finally, statistical properties of fluctuating density measurements in the SOL of the TCV tokamak are compared with those associated with density, temperature and potential fluctuations obtained in a particular TORPEX plasma scenario, characterized by strong similarity to a tokamak SOL (section 7).

\section{Experimental setup}

The experiments are performed in TORPEX, a toroidal device with major and minor radius $R=1 \mathrm{~m}$ and $a=0.2 \mathrm{~m}$ and a toroidal magnetic field up to $0.1 \mathrm{~T}$. A small vertical magnetic field component $\leqslant 4 \mathrm{mT}$ is superimposed to partly short circuit the vertical electric field due to $\nabla B$ and curvature drifts and reduce the particle losses [6]. Highly reproducible plasmas are created and sustained for up to $3 \mathrm{~s}$ by means of waves in the electron cyclotron range of frequencies [7]. Measurements of ion saturation current are obtained over the whole plasma cross-section (comprising ionization source, edge and confinement region) in a single plasma discharge, with a spatial resolution of $3.5 \mathrm{~cm}$ and at a sampling frequency of $250 \mathrm{kHz}$ with a hexagonal array of 86 Langmuir probes, named HEXTIP [8]. As in these experiments temperature fluctuations are small compared with density fluctuations, ion saturation current fluctuations are assumed to be directly proportional to electron density fluctuations. The microwave power and the toroidal magnetic field are kept constant. Different neutral gases are used $(\mathrm{H}, \mathrm{He}, \mathrm{Ar})$; the vertical magnetic field and the neutral gas pressure are varied, leading to variations of the ion gyroradius, the plasma shape and the ion-neutral collision frequency. As a consequence, for all signals the range of time averaged densities $\bar{n}$ and electron temperature $\bar{T}_{\mathrm{e}}$ extends between $1.4 \times 10^{15} \mathrm{~m}^{-3}$ and $3.7 \times 10^{17} \mathrm{~m}^{-3}$ and $1 \mathrm{eV}$ and $10 \mathrm{eV}$, respectively. The range of fluctuation levels is $1 \% \leqslant \delta n / \bar{n}<95 \%$, where $\delta n$ is the rms fluctuating density. The observed density fluctuations are associated with drift-interchange instabilities, generated in regions of bad field curvature and convected by the $E \times B$ fluid motion [5,9]. The nature of these fluctuations is relevant for edge/SOL turbulence studies. Note that the achieved range of density fluctuations is large compared with what can be covered in tokamaks, even across several different devices. 


\section{Local statistical properties and relation with coherent modes and structure trajectories}

In this section, we focus on a subset of the data which corresponds to a neutral gas pressure scan in argon. To obtain the PDF of the plasma density across the plasma cross-section, the ion saturation current $I_{\text {sat }}$ measured by each tip of HEXTIP is binned. Without an a priori idea on the PDF shape, a good compromise between resolution in the binned quantity and statistics is given by choosing the number of bins $N_{\text {bin }}=\mathcal{O}(\sqrt{N})$ ( $N$ is the number of points in the time series). As the binning introduces some noise on the distribution, we choose to smooth the PDF, using a smoothing parameter fixed to $\sqrt{N} / 10$.

Five analytical functions, frequently used in plasma physics (normal, gamma, log-normal, extreme value, Gumbel) are considered for describing the experimental distributions. All of them have two free parameters, the mean value $\mu$ and the variance $\sigma^{2}$, and are normalized in such a way that $\int_{-\infty}^{+\infty} f(x) \mathrm{d} x=1$. To determine which distribution provides the best fit to the experimental data, two different approaches are followed: to insert the parameters $\mu_{\exp }$ and $\sigma_{\text {exp }}$ estimated from the measured signals in the analytical expressions or to let them free to evolve in order to minimize the residual. The quality of a fit is estimated by comparing the different residuals:

$$
\begin{aligned}
& s=\sum_{i=1}^{N_{\text {bin }}}\left[f^{\text {smooth }}\left(x_{i}\right)-f\left(x_{i}\right)\right]^{2}, \\
& s_{\text {exp }}=\sum_{i=1}^{N_{\text {bin }}}\left[f^{\text {smooth }}\left(x_{i}\right)-f^{\text {exp }}\left(x_{i}\right)\right]^{2}, \\
& s_{\text {opt }}=\sum_{i=1}^{N_{\text {bin }}}\left[f^{\text {smooth }}\left(x_{i}\right)-f^{\text {opt }}\left(x_{i}\right)\right]^{2},
\end{aligned}
$$

where $f$ is the binned distribution from experimental signal, $f^{\text {smooth }}$ is the smoothed experimental distribution, $f^{\exp }$ an analytical distribution with $\mu_{\exp }$ and $\sigma_{\text {exp }}$ and $f^{\text {opt }}$ an analytical distribution with the optimal mean value and variance. A good fit corresponds to $s_{\text {exp,opt }} \leqslant s$.

The result for the fit with the analytical distributions evaluated with $\mu_{\mathrm{opt}}$ and $\sigma_{\mathrm{opt}}$ is plotted on figure 1(a) for three selected neutral gas pressure values. Results for a more complete pressure scan are presented in [10]. For all the neutral gas pressure values, double-humped PDFs (dark gray hexagons) are present. A doubled-humped distribution is associated with large amplitude coherent modes. This is confirmed by the correlation between the presence of a double-humped distribution and a large ratio of the energy contained in the coherent modes to the total energy (figure $1(b)$ ). Coherent modes have been identified from the statistical frequency distribution computed from the spectra of the 86 tips of HEXTIP [9]. The range of frequencies over which the spectra are integrated to estimate the energy is given by the full-width at half-maximum of the distribution.

At high pressure, almost all single-humped distributions can be fitted by any of the tested PDFs (white hexagons). In contrast, at low pressure, some measured distributions corresponding roughly to the transition between double-humped and single-humped can be fitted by none of the tested distributions (black hexagons). Finally, for a small subset of the experimental data, we find that only one tested PDF yields a good fit (soft gray hexagons). In this case, one can conclude that none of the analytical functions are predominantly present compared with the others and that no particular spatial localization or particular value of the 

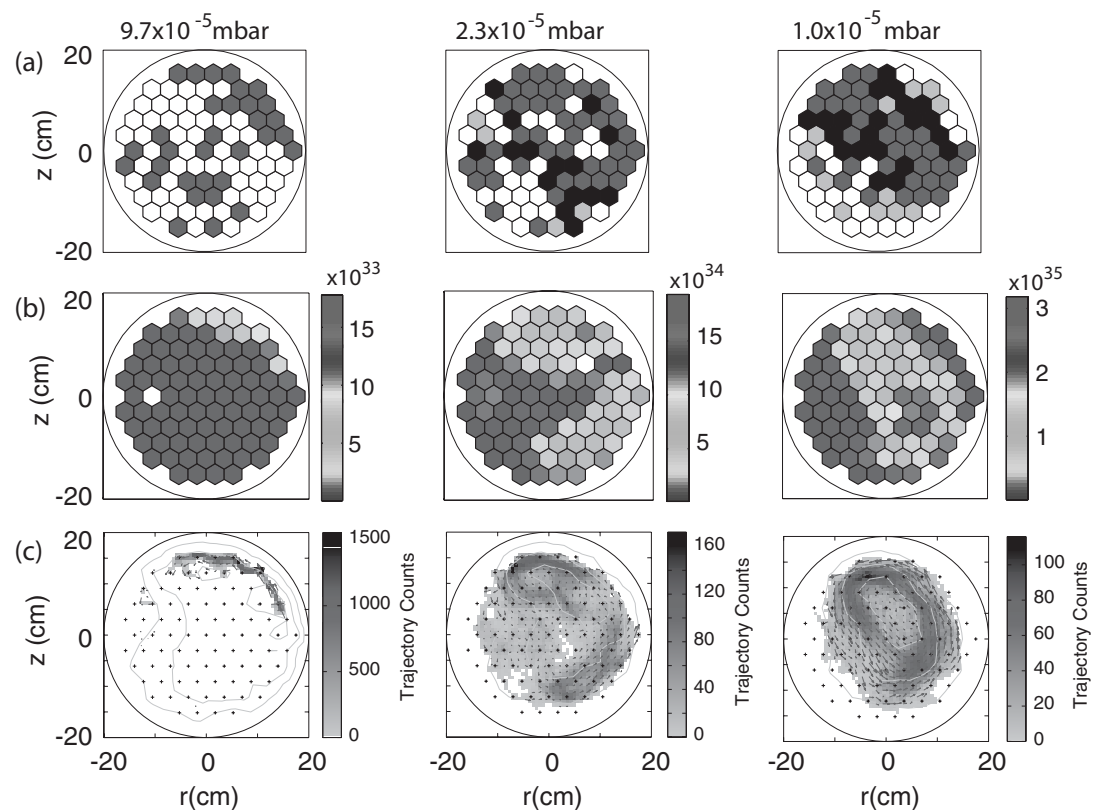

Figure 1. For three different neutral gas pressures: $(a)$ result of the fit with optimized parameters. The color coding is the following: several PDF fit the experimental one (white), no PDF fit the experimental one (black), only one PDF fits the experimental one (soft gray), double-humped PDF (dark gray); (b) spectral energy contained in the mode and (c) statistics of structure trajectories.

neutral gas pressure is correlated with a given analytical distribution [10]. In figure 1(c) we represent the trajectories of the fluctuation structures. Structures are defined as spatial regions where the density deviation on average exceeds a threshold value. By following these structures in time, their trajectories can be identified [11]. It is clear that the modes with large amplitude and the trajectories associated with a drift-wave propagating with the $\boldsymbol{E} \times \boldsymbol{B}$ velocity are located where an experimental time series is characterized by a double-humped distribution.

\section{Unique relation between skewness and kurtosis}

A fitting method based on the 1st and 2nd moments of the PDF cannot provide a clear evidence of universality for the statistical properties of density fluctuations. A new approach based on higher moments is presented in this section. For around 10000 HEXTIP signals, the skewness (normalized third order moment of the PDF) and the kurtosis (normalized fourth order moment of the PDF) are estimated and plotted against each other (figure 2). We observe that a significant fraction of the signals $(\simeq 30 \%)$ are characterized by a negative skewness. Previous measurements in tokamak plasmas [12] reported a change in the skewness sign from positive to negative values when crossing the last closed flux surface from the SOL region into the plasma edge. The data in figure 2 are least-square fitted by the quadratic polynomial

$$
K=(1.502 \pm 0.015) S^{2}+(2.784 \pm 0.019)
$$

The uncertainty on the estimated coefficients corresponds to the $95 \%$ confidence bounds. This interval is illustrated on the graph with error bars. The inset in figure 2 shows that approximately 


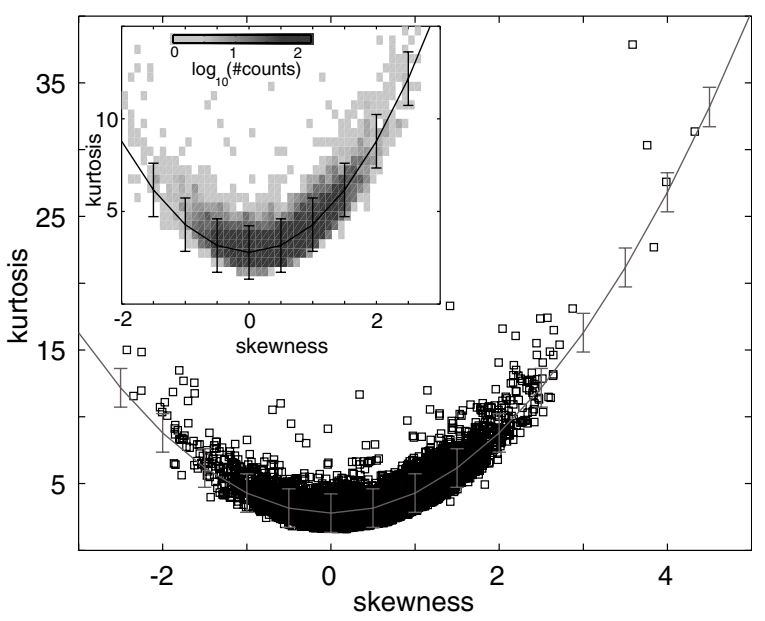

Figure 2. Kurtosis versus skewness computed for 8966 experimental signals. The fitting second order polynomial (solid grey line) is also plotted. (Inset) Joint probability of $K$ and $S$ for the experimental signals. On both graphs, the error bars correspond to the $95 \%$ confidence interval.

95\% of the experimental data is contained within these error bars. Such a link between third and fourth order moments is valid for a large set of PDF families [13]. This emphasizes the importance of identifying a particular parabolic relation when trying to determine a theoretical PDF that reproduces experimental distributions. Because of the functional relation between the kurtosis and the skewness, none of the analytical PDFs that are defined with constant $S$ and $K$ (normal, logistic, Rayleigh, uniform, exponential, etc) can universally reproduce the experimental measurements. Furthermore, a quadratic polynomial rules out distributions (triangular, error, Student's, etc) for which either $K$ or $S$ varies, but the other moment is constant. Finally, the robustness of the least-square fit (2) excludes distributions (Weibull, log-normal, Wald, $F$, etc) that have a similar relationship between $K$ and $S$ but with different coefficients [13]. All these PDFs can be excluded together with the underlying physical basis from which they originate. The question one needs to answer now is: is there an analytical distribution that is characterized by a relationship between its skewness and its kurtosis identical to the one found from the experiment?

\section{The beta distribution and its application to experimental results}

The polynomial in (2) is very close to the relation between the kurtosis and the skewness associated with the gamma distribution: $K=1.5 S^{2}+3$, although the skewness of the gamma distribution is by definition always positive [13]. One distribution that can admit both positive and negative values for the skewness is the beta distribution [13]. For a random variable $n$, the general beta distribution is defined by

$$
F_{\beta}\left(n ; p, q, n_{l}, n_{h}\right)=\frac{\left(n-n_{l}\right)^{p-1}\left(n_{h}-n\right)^{q-1}}{\mathcal{B}(p, q)\left(n_{h}-n_{l}\right)^{p+q-1}},
$$

where $n_{l} \leqslant n \leqslant n_{h} ; p, q>0$ and $\mathcal{B}$ is the beta function defined as $\mathcal{B}(p, q)=$ $\int_{0}^{1} t^{p-1}(1-t)^{q-1} \mathrm{~d} t$. Its first four normalized moments can be computed from

$$
\mu=\frac{p}{p+q}\left(n_{h}-n_{l}\right)+n_{l},
$$




$$
\begin{aligned}
\sigma^{2} & =\frac{p q}{(p+q)^{2}(p+q+1)}\left(n_{h}-n_{l}\right)^{2}, \\
S & =\frac{2(q-p)}{(p+q+2)} \sqrt{\frac{p+q+1}{p q}}
\end{aligned}
$$

and

$$
K=\frac{3(p+q+1)\left[2(p+q)^{2}+p q(p+q-6)\right]}{p q(p+q+2)(p+q+3)} .
$$

The relation between the third and fourth moments is not trivial since it depends on parameters $p$ and $q$, but $S$ and $K$ do not depend explicitly on the boundaries of the random variable $n$. Moreover, for any $(p, q)$ it can be shown that [13]

$$
1+S^{2}(p, q) \leqslant K(p, q) \leqslant 3+1.5 S^{2}(p, q) .
$$

The upper limit is reached when $q \rightarrow+\infty$ for positive skewness and when $p \rightarrow+\infty$ for negative skewness. The experimentally measured skewness and kurtosis are very close to the upper limit of the domain of variation for a beta distribution; thus either $p$ or $q$ has to be large. In [4], we have shown that the best choice for the boundaries $n_{l}$ and $n_{h}$ is to take $n_{l}=n_{\min }$ and $n_{h}=n_{\max }$ where $n_{\min }$ and $n_{\max }$ are the minimal and the maximal density for each time trace, respectively. With this choice, one can estimate parameters $p$ and $q$ from (4)-(5): $p=\bar{\mu}\left[\bar{\mu}(1-\bar{\mu}) / \bar{\sigma}^{2}-1\right], q=(1-\bar{\mu})\left[\bar{\mu}(1-\bar{\mu}) / \bar{\sigma}^{2}-1\right]$ with $\bar{\mu}=\left(\mu_{\exp }-n_{\min }\right) /\left(n_{\max }-n_{\min }\right)$ and $\bar{\sigma}^{2}=\sigma_{\exp }^{2} /\left[\left(n_{\max }-n_{\min }\right)^{2}\right]$. Since typical values for $p$ and $q$ are larger than 100, $S$ and $K$ for a beta distribution (6)-(7) are close to the upper limit in (8) and are compatible with the experimental third and fourth moments.

An illustration of the good agreement between the experimental PDF and the beta distribution is shown in figures $3(a)$ and $(b)$ for negative and positive skewed experimental distributions. Table 1 summarizes the main parameters associated with these two experimental distributions. For all the signals, we repeat the analysis presented in section 3. We compute the residual $s_{\exp }$ between the experimental distribution and several analytical PDFs, including now the beta distribution, evaluated with $\mu_{\exp }$ and $\sigma_{\exp }$. The PDF of the residuals is shown in figure 3(c). The beta distribution provides the smallest average residual as well as a better fit than any other tested PDF in the region of small residuals $\left(s_{\exp } \leqslant 0.1\right)$. All the experimental PDFs can be universally reproduced by a limiting case of the beta distribution.

\section{Role of the drift-interchange frequency range}

To address the question of which physical mechanisms contribute to such a behavior, we investigate which frequency range of the fluctuations spectrum, if any, is necessary and sufficient to recover the beta distribution. For the three neutral gases used, $M \simeq 800$ signals have been filtered at different cut-off frequencies $\left(0.1 \mathrm{kHz} \leqslant f_{c} \leqslant 50 \mathrm{kHz}\right)$ with both low-pass and high-pass filters. For all the cut-off frequencies $f_{c}$, the skewness $S_{j}\left(f_{c}\right)$ and the kurtosis $K_{j}\left(f_{c}\right)$ of the filtered signals are estimated. For each $f_{c}$, the residual defined by

$$
r_{f_{c}}=\sum_{j=1}^{M}\left[K_{j}\left(f_{c}\right)-\left(1.502 S_{j}\left(f_{c}\right)^{2}+2.784\right)\right]^{2}
$$

is computed and normalized to the original residual for the unfiltered signals,

$$
r_{\exp }=\sum_{j=1}^{M}\left[K_{j, \exp }-\left(1.502 S_{j, \exp }^{2}+2.784\right)\right]^{2} .
$$



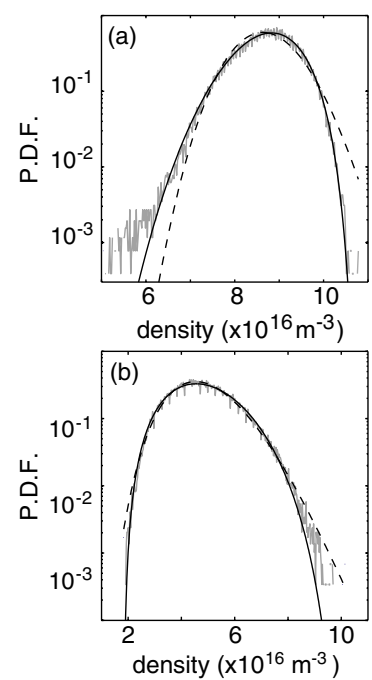
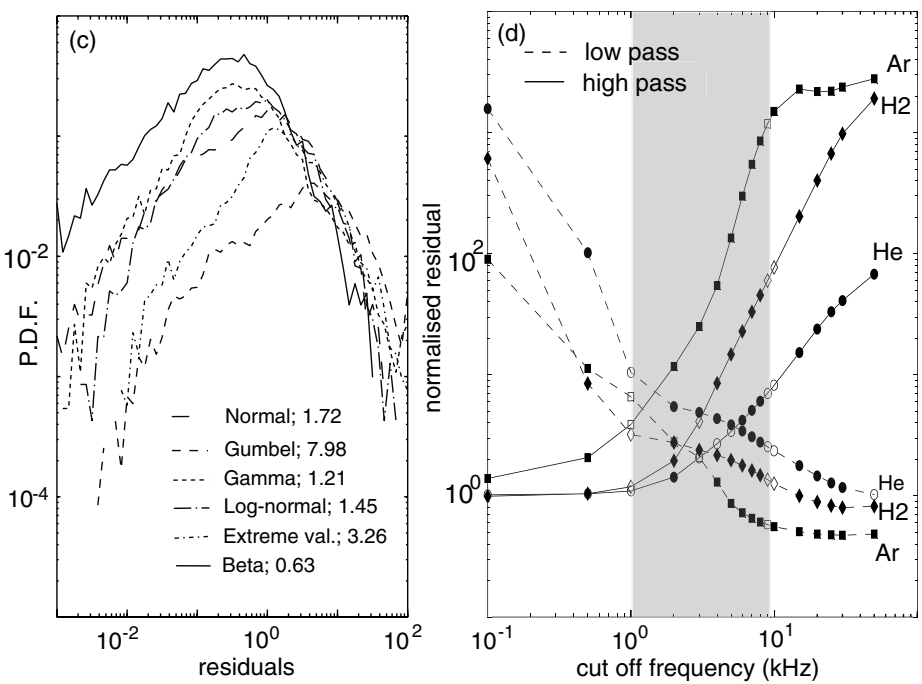

Figure 3. (a) and (b) Two experimental distributions from argon plasmas (gray line) with a negative (a) and positive $(b)$ skewness. The beta distribution (solid) and the gamma distribution (dashed) estimated with $\mu_{\exp }$ and $\sigma_{\exp }$ are also plotted. (c) Distributions of the residuals between all the experimental PDFs and several analytical distributions estimated with $\mu_{\exp }$ and $\sigma_{\text {exp }}$. The average value of the residuals is indicated in the legend. $(d)$ Normalized residuals for different cut-off frequencies of a low-pass and a high-pass filters applied to experimental signals. The shadowed region represents the drift-interchange frequency range.

Table 1. Numerical values of experimental parameters associated with the PDFs plotted in figures $3(a)$ and $(b)$. $\mu_{\exp }, \sigma_{\exp }, n_{\min }$ and $n_{\max }$ are normalized to $10^{16}$. Parameters $s, s_{\beta}$ and $s_{\Gamma}$ are different residuals computed from equations $(1 a)$ and $(1 b)$.

\begin{tabular}{lllllll}
\hline & $\mu_{\exp }$ & $\sigma_{\exp }$ & $S_{\exp }$ & $K_{\exp }$ & $n_{\min }$ & $n_{\max }$ \\
\hline Figure 3 $(a)$ & 8.68 & 0.67 & -0.46 & 3.51 & 3.48 & 10.79 \\
Figure 3(b) & 4.81 & 1.16 & 0.41 & 2.92 & 1.82 & 10.15 \\
& $p$ & $q$ & $s$ & $s_{\beta}$ & $s_{\Gamma}$ & \\
\hline Figure 3(a) & 16.78 & 6.79 & 0.39 & 0.08 & 0.33 & \\
Figure 3(b) & 3.92 & 7.00 & 0.10 & 0.01 & 0.04 & \\
\hline
\end{tabular}

This quantity is represented in figure $3(d)$ for different cut-off frequencies and for the three gases. As $f_{c}$ increases (decreases) for the low-pass (high-pass) filters, the difference between the original moments and those associated with filtered signals tends to disappear. For the three gases, if only the low $\left(f_{c} \leqslant 1 \mathrm{kHz}\right)$ or high $\left(f_{c} \geqslant 20 \mathrm{kHz}\right)$ frequency contributions to fluctuations are kept, the experimental fit described by (2) cannot be satisfied. The density fluctuations contained in the measured frequency range of drift-wave instabilities $(1 \mathrm{kHz} \leqslant f \leqslant 10 \mathrm{kHz}$ ) [9] must be retained to recover this beta distribution.

\section{Statistical properties of SOL-like plasmas in TORPEX}

Up to now, only the statistical properties of the plasma density fluctuations in TORPEX have been investigated. In this section, we report on similar studies for density measurements from other toroidal plasmas, the SOL plasmas of the TCV tokamak, but also for quantities other than the density, such as the electron temperature and the plasma potential measured on TORPEX. 

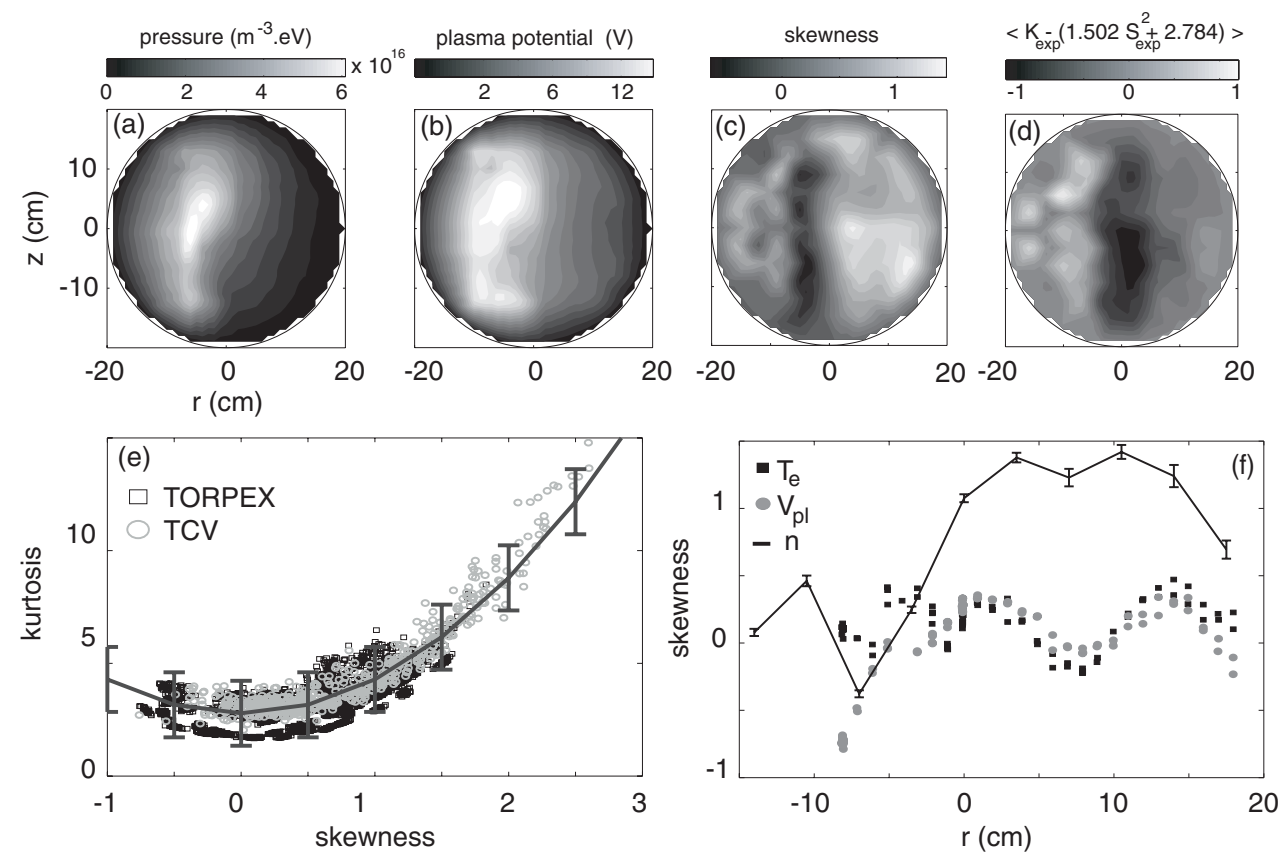

Figure 4. The 2D poloidal profiles of the time-averaged plasma pressure $(a)$, plasma potential $(b)$, density fluctuations skewness $(c)$ and $(d)$ difference between the experimental kurtosis and values from (2) (e). Kurtosis versus skewness for 2580 signals in the 'SOL-like configuration' in TORPEX (squares) and for 962 signals measured in the TCV SOL. $(f)$ Radial profiles of the skewness estimated for $n, T_{\mathrm{e}}$ and $V_{\mathrm{pl}}$.

Measurements of electron temperature fluctuations and plasma potential fluctuations on TORPEX have been performed in a particular plasma scenario. Hydrogen plasmas are produced by microwaves with $P_{\mathrm{rf}} \simeq 400 \mathrm{~W}$. The plasma source is localized on the highfield side, with negligible plasma production for $r \geqslant 5 \mathrm{~cm}$. By using a relatively high vertical magnetic field $\left(B_{\varphi}=76 \mathrm{mT}, B_{z}=2.3 \mathrm{mT}\right)$, the profiles of pressure and plasma potential are vertically elongated, peak in the high field side region and decrease towards the low field side (figures $4(a)$ and $(b)$ ). The poloidal profile of the skewness estimated for the 86 signals measured by HEXTIP and averaged over 30 similar shots is plotted in figure $4(c)$. In this configuration, we identify two distinct poloidal regions with different plasma dynamics: (1) a main plasma region for $r \leqslant 5 \mathrm{~cm}$ dominated by a coherent interchange mode at $f \simeq 4 \mathrm{kHz}$. The inner part of this region is characterized by density fluctuations with negative skewness (figure 4(c)); (2) a region on the low field side for $r \geqslant 5 \mathrm{~cm}$ with negligible plasma production, dubbed a source-free region (as no plasma production process is present), characterized by the propagation of plasma blobs leading to positively skewed PDF and resulting in intermittent transport events [14]. The mechanism for plasma blob formation in TORPEX has been investigated using time-resolved measurements of two-dimensional structures of electron density, temperature and plasma potential. The blobs are observed to form from a radially elongated structure that is sheared off by the $\boldsymbol{E} \times \boldsymbol{B}$ flow [15].

The ensemble average difference between the experimental kurtosis and the values obtained from (2) with the experimental skewness is plotted in figure $4(d)$. The agreement is good for fluctuations in the source-free region. The mode region is characterized by double-humped PDF, with kurtosis lower than 3 (the value associated with the Gaussian 
distribution) and so experimental kurtosis is not perfectly fitted by (2). Nevertheless, the relationship between the skewness and the kurtosis for these signals is still parabolic (figure $4(e)$ ) and lies within the error bars provided by the $95 \%$ confidence bounds on the polynomial coefficients.

Figure 4(e) also shows the kurtosis versus the skewness for plasma density measurements taken with a reciprocating Langmuir probe at different radial locations in the edge/SOL of the TCV tokamak. The experimental values, corresponding to $24 \mathrm{~L}$-mode diverted plasmas in different conditions $\left(I_{\mathrm{p}} \gtrless 0,3.2 \times 10^{19} \leqslant \bar{n}_{\mathrm{e}} \leqslant 12 \times 10^{19} \mathrm{~m}^{-3}\right.$, gas: He, De) [16], are well fitted by (2). For SOL signals characterized by positive skewness, the gamma distribution has been previously reported to be compatible with their PDF [17]. But indeed, for large parameters $p$ and $q$, the beta distribution is equivalent to $F_{\Gamma}(n)$ for positive skewness and to $F_{\Gamma}(2 \mu-n)$ for negative skewness, where $\mu$ is the mean value of the density $n$ and $F_{\Gamma}$ is the gamma distribution defined as $f_{\Gamma}(n ; \alpha, \theta)=\theta^{-\alpha} n^{\alpha-1} \mathrm{e}^{-n / \theta} / \Gamma(\alpha)$ with $\Gamma(\alpha)=\int_{0}^{\infty} t^{\alpha-1} \mathrm{e}^{-t} \mathrm{~d} t$ is the gamma function. In a sense, this extends the previous results for the TCV tokamak to negatively skewed PDFs mainly associated with signals measured inside the LCFS. Similarly to TORPEX plasmas described before, the interchange turbulence plays a prominent role at the edge/SOL boundary layer, confirmed by numerical simulations [18].

Finally, we address the question of statistical properties of quantities other than the plasma density. The radial profiles of the skewness of the electron temperature and of the plasma potential are shown in figure $4(f)$. The time evolution of $T_{\mathrm{e}}$ and $V_{\mathrm{pl}}$ is measured using a triple probe [19]. The statistical properties of these quantities are much more Gaussian than for the density fluctuations. This is due to the fact that, in this case, $\delta T_{\mathrm{e}} / T_{\mathrm{e}} \simeq \delta V_{\mathrm{pl}} / V_{\mathrm{pl}} \leqslant 10 \%$ while $20 \% \leqslant \delta n / n \leqslant 60 \%$. This suggests that there is some heat transport associated with a plasma blob but this loss channel is much less efficient than the heat transport associated with the electrons moving fast along the field lines [19].

\section{Conclusion and outlook}

We have analyzed electrostatic fluctuations measured over the entire cross-section of a simple toroidal magnetized plasma, including the source region for instabilities, regions where nonlinear mode coupling takes place and regions where the unstable modes are convected by the $E \times B$ flow [20]. For a subset of experimental data we have shown that double-humped distributions are localized at the same position as the trajectories and the largest amplitude of the coherent modes. This constitutes a first attempt at linking spectral features, spatio-temporal evolution and statistical properties of fluctuations in a toroidal plasma. Moreover, the singlehumped PDFs of the density fluctuations at all locations are found to have a universal character, in the sense that a unique relation links the skewness and the kurtosis and that it can be described by the same analytical distribution. This is a special case of the general beta distribution $F_{\beta}(x)$, taking the form of the gamma distribution $F_{\Gamma}$, depending on the sign of the signal skewness. This specific Beta distribution can also reproduce density fluctuation signals, including those with negatively skewed PDF, measured in the SOL of tokamaks. These observations are consistent with the picture of a unique physical mechanism producing events that would generate in the same region positive and negative density structures, corresponding to a local excess of density and a density depletion, respectively [11,21]. Both kinds of structures would carry along their motion the same PDF features, as they originate from the same local event. Only the fluctuations in the typical range of frequency associated with drift-wave turbulence are necessary to assure that PDFs can be described by this specific beta distribution, ruling out an interpretation of the fluctuations statistical properties resulting from a fully developed turbulence. A more complete physical understanding of plasma turbulence in the present 
experiments requires extensive self-consistent numerical simulations of drift-interchange turbulence using nonlinear equations, e.g. based on the Hasegawa-Wakatani model. Such simulations are currently run for TORPEX plasmas. Nevertheless, the present results may have two consequences on the study of the underlying physics: the unique relation between moments of the plasma density PDF may lead to a low-order statistical closure of the chain of coupled equations [2]; the same global characterization of the statistical properties of various fluctuating fields, applied to numerical simulation outputs, may provide a valuable tool for the validation of the physical basis of the simulation model.

\section{Acknowledgments}

This work is partly funded by the Fonds National Suisse de la Recherche Scientifique. Fruitful discussions with Professor F Skiff, Dr R Pitts and Dr J P Graves are acknowledged. PR and JH are supported by a EURATOM Fusion fellowship.

\section{References}

[1] Horton W 1990 Phys. Rep. 1921

[2] Krommes J A 2002 Phys. Rep. 3601

[3] Frisch U 1995 Turbulence, The Legacy of A. N. Kolmogorov (Cambridge: Cambridge University Press)

[4] Labit B et al 2007 Phys. Rev. Lett. 98255002

[5] Fasoli A et al 2006 Phys. Plasmas 13055903

[6] Müller S H et al 2004 Phys. Rev. Lett. 93165003

[7] Podestà M et al 2005 Plasma Phys. Control. Fusion 471989 Podestà M et al 2006 Plasma Phys. Control. Fusion 481053

[8] Müller S H et al 2005 Phys. Plasmas 12090906

[9] Poli F M et al 2006 Phys. Plasmas 13102104

[10] Labit B et al 2006 Proc. 33rd EPS Conf. on Controlled Fusion and Plasma Physics (Rome, 19-23 June 2006) ed F De Marco and G Vlad vol 30I (ECA) p1.143

[11] Müller S H et al 2006 Phys. Plasmas 13100701

[12] Boedo J A et al 2003 Phys. Plasmas 101670

[13] Johnson N L, Kotz S and Balakrishnan N 1995 Continuous Univariate Distributions 2nd edn (New York: Wiley)

[14] Müller S H 2006 Turbulence in basic toroidal plasmas PhD Thesis No 3672 École Polytechnique Fédérale de Lausanne http://library.epfl.ch/theses/?nr=3672

[15] Furno I et al 2007 Mechanism for plasma blob generation from drift-interchange waves Phys. Rev. Lett. submitted

[16] Horacek J 2006 Measurements of edge electrostatic turbulence in the TCV tokamak plasma boundary PhD Thesis No 3524 École Polytechnique Fédérale de Lausanne http://library.epfl.ch/theses/?nr=3524

[17] Graves J P et al 2005 Plasma Phys. Control. Fusion 47 L1

[18] Garcia O E et al 2006 Plasma Phys. Control. Fusion 48 L1 Garcia O E et al 2004 Phys. Rev. Lett. 92165003

[19] Podestà M 2007 Plasma production and transport in a simple magnetised toroidal plasma PhD Thesis No 3765 École Polytechnique Fédérale de Lausanne http://library.epfl.ch/theses/?nr=3765

[20] Poli F M, Podestà M and Fasoli A 2007 Phys. Plasmas 14052311

[21] Carter T A 2006 Phys. Plasmas 13010701 